\title{
Pathologising trans people: Exploring the roles of patients and medical personnel
}

\author{
Kinnon Ross MacKinnon ${ }^{1}$
}

\begin{abstract}
Despite the 2013 decision to replace the diagnostic labelling of gender identity disorder with gender dysphoria, there remains wide consensus amongst scholars that transgender identities are pathologised. Yet the claim that trans identity has been pathologised has yet to be rigorously substantiated. Adapting a model for verifying the credibility of medicalisation claims, this inquiry confirms that the human phenomenon of gender-variance has underwent pathologisation to embed trans identity within a medical model by (1) historicising how trans identity became viewed as a medical condition; (2) highlighting that medical explanations for trans identity are adopted by trans people and the culture at large; and (3) showing that medical theory for trans identity gains traction over any other explanation. [Article copies available for a fee from The Transformative Studies Institute. E-mail address: journal@transformativestudies.org Website: $\quad$ http://www.transformativestudies.org 02018 by The Transformative Studies Institute. All rights reserved.]
\end{abstract}

KEYWORDS: Transgender, Trans, Gender Dysphoria, Medicalisation, Pathologisation.

\section{INTRODUCTION}

In contemporary Western nations most people identify and express their gender in ways that culturally match their birth sex. These people can be described as cisgender and are contentiously understood to represent the baseline of 'healthy' or 'normal' gender identity and expression, despite significant rejection of these labels from transgender health research and advocacy (Davis et al. 2016; Schilt \& Westbrook 2009). On the other hand transgender and other gender-variant (trans) people experience a

\footnotetext{
${ }^{1}$ Kinnon Ross MacKinnon, MSW, is a $\mathrm{PhD}$ candidate in public health at the University of Toronto and a fellow with the Wilson Centre at University Health Network. Address correspondence to: Kinnon Ross MacKinnon, University of Toronto, Dalla Lana School of Public Health, 155 College Street, Toronto M5T3M7, Canada; e-mail:

k.mackinnon@mail.utoronto.ca.
} 